\title{
Using CNN's Gait Recognition to Strengthen Laboratory Safety Supervision
}

\author{
Yongjia Xu ${ }^{1}$, Fuji Ren ${ }^{1+}$ and Shun Nishide ${ }^{1}$ \\ ${ }^{1}$ Faculty of Engineering, Tokushima University 2-1 Minami Josanjima, Tokushima, 770-8506, Japan
}

\begin{abstract}
In addition to manual management, the security measures of important facilities such as school laboratories mainly rely on human body recognition systems. Gait recognition is non-intrusive, the identification process is fast and simple, and the recognition method is less affected by clothes, which makes it suitable for laboratory's safety monitoring purposes. This paper uses the form of gait energy image to extract the gait information features of the human body. The main contribution of the paper is the improvement of existing CNN models, adding a batch-normalization layer to for better recognition. Two types of experiments were conducted. The first experiment is a comparison with previous methods using the CASIA standard dataset. The second experiment is the evaluation with our new dataset using OpenCV to collect gait information in an actual laboratory environment. The results of the experiment show that the proposed method and its application in a real environment are feasible to enhance laboratory safety monitoring.
\end{abstract}

Keywords: gait recognition, CNN, deep learning, OpenCV

\section{Introduction}

At present, university computer laboratories are one of the key teaching and research places related to computers and other related fields. These laboratories contain expensive and valuable equipment requiring high information security and safety management. The mainstream security management measures for most laboratories are mainly divided into two categories; one is to arrange the laboratory administrator for supervision work, and the other is to install face recognition, fingerprint recognition and another feature verification device on the laboratory door [1][2][3]. However, even if the laboratory has implemented these regulatory measures, there are still some loopholes in the security aspect. For example, the biological information of a supervision personnel, such as face and fingerprints, could easily be forged [4][5][6]. In order to further improve the security level of computer labs, this paper proposes to add a camera on the road to the laboratory, and use the gait recognition method to identify the visitors [7][8]. This adds a layer of gait information recognition system to the traditional access control security, which further improves the security management model of the entire computer lab. The advantages and drawbacks of applying gait recognition are explained in the following subsections.

\subsection{Characteristics and advantages of gait recognition}

Biometric identification refers to the use of physical behavior to identify humans [9]. Gait recognition is a biometric technology that determines an individual's identity based on each person's different walking styles. In recent years, the technology of extracting human gait information from a video has achieved a staged breakthrough. Regardless of the method to extract information, whether it be human body modeling or masking, it is possible to extract the amount of gait information from one or several consecutive frames of video [10][11].

Corresponding author. Tel.: + 81886569684; fax: +81-88-656-6575.

E-mail address: ren@is.tokushima-u.ac.jp 
Gait recognition has the following advantages over other biometric technologies: 1. Non-intrusive identification that does not influence other objects nor does it require the body to make direct contact with any sensor. The recognition process is simple and unnoticeable by the subject. 2 . The monitoring system is not required to be close to the subject, does not require high resolution, and the extracted feature video is easy to obtain. 3. Compared with other biometric methods such as face, fingerprint, and voiceprint, gait recognition is not disturbed by masks, sunglasses, and any other type of clothes [11][12]. Thus, human gait features are difficult to forge compared with other biometric features. Under certain conditions, the recognition result of gait recognition could be more reliable. This paper prepares to build a set of safety management system that collects and identifies human gait information by using gait recognition technology.

\section{Related Work}

In this section, we introduce several gait recognition methods that have been worked on, and describe the positioning of our study. The following two major contributions presented in this paper are introduced.

\subsection{Improvement of CNN model}

Gait Energy Image (GEI) is one of the main methods for quantifying gait feature for deep learning. Convolutional Neural Networks (CNN) are currently a mainstream method for deep learning. For example, Shirage et al. utilized a CNN consisting of a convolutional layer, a pooling layer, a normalization layer, two consecutive triples, and two subsequent fully-coupled layers for gait recognition [12]. They used GEI features to obtain good results of gait recognition. Alotaibi et al. constructed a self-developed DEEP CNN network, and used this network to train and classify the GEI features in CASIA DatasetB, and obtained considerable results [14]. Comparing with existing Deep CNNs, this paper adds a Batch Normalization layer to the original structure of the model, which further improves the recognition result.

\subsection{Establish gait data sets for laboratory personnel}

There are many GEI open datasets available for research today, such as CASIA's large multiview gait dataset CASIA Dataset B and the OU-ISIR large population dataset [15]. Both of these datasets provide a variety of GEI images under various clothing conditions from multiple viewing angles for researchers to study. In this paper, laboratory members were recruited as volunteers to collect gait data in the form of the two datasets, and multiple sets of GEI data corresponding to each volunteer were obtained. The data were tagged and classified according to the same format of the two datasets for training and classification by the proposed CNN model.

\section{Component Technologies of the Proposed Method}

This section introduces the component technologies used to construct the model. The contents are divided into three parts: 1. Gait Energy Image (GEI), 2. Convolutional Neural Network (CNN), and 3. Dataset.

\subsection{Gait Energy Image (GEI)}

We apply the Gait Energy Image (GEI) proposed by Han et al. for extracting gait information [16].

First, the gray level GEI is defined as

$$
G(x, y)=\frac{1}{N} \sum_{t=1}^{N} B_{t}(x, y)
$$

where $\mathrm{N}$ is the number of frames in the period and $(\mathrm{x}, \mathrm{y})$ is the specific coordinate value. This formula has three characteristics: 1. Each frame silhouette represents the energy image of the person walking at the moment. 2. A GEI image is an energy image that a pedestrian fully accumulates in a gait cycle. 3. In the GEI diagram, a high intensity value of a coordinate results in a higher gait energy of the pedestrian [16][17][18].

\subsection{Convolutional neural network}

The convolutional neural network, $\mathrm{CNN}$, is one of the main networks for image classification and recognition. CNN can be utilized for deep object learning, object detection recognition, and the like [19]. 
Recently, Deep CNNs have many applications in the image field. For example, Zhang et al. introduced an image denoising task using DnCNN model [20]. Chen et al. proposed an unconstrained algorithm using Deep CNN for feature-based human connection verification, obtaining good experimental results [21]. Their paper also refers to the model by Alotaibi et al, using a self-made Deep CNN architecture to complete the identification of the CASIA gait database with different viewing angles. In the comparison experiment of single identification and cross recognition, their method proved to obtain good experimental results [14].

As shown in these papers, CNNs, specifically Deep CNNs, have a good application advantage in image processing and recognition, including the field of gait recognition. In this paper, we construct a serial CNN network for classification and identification using GEI datasets.

\subsection{Dataset}

Among various GEI datasets, we adopt CASIA Dataset B, a large multi-view gait dataset created by the Institute of Automation of Chinese Academy of Sciences. The model was evaluated with a test dataset, and then the Dataset B was created using the student's own gait dataset [22]. CASIA-B is a large-scale multi-view gait library with 124 people in the dataset, each from 11 perspectives $(0,18,36,54,72,90,108$, 126, 144 and 180 degrees). Data were collected under three kinds of walking conditions (common conditions, wearing coats, carrying parcel conditions).

\section{Construction of the Proposed CNN Model}

The proposed Deep CNN model is based on the model used by Alotaibi et al. [14][23], which comprises 8 layers: 4 convolution layers and 4 pooling layers with 8 feature maps in each layer. The CNN architecture by Alotaibi et al. is shown in Fig. 1.

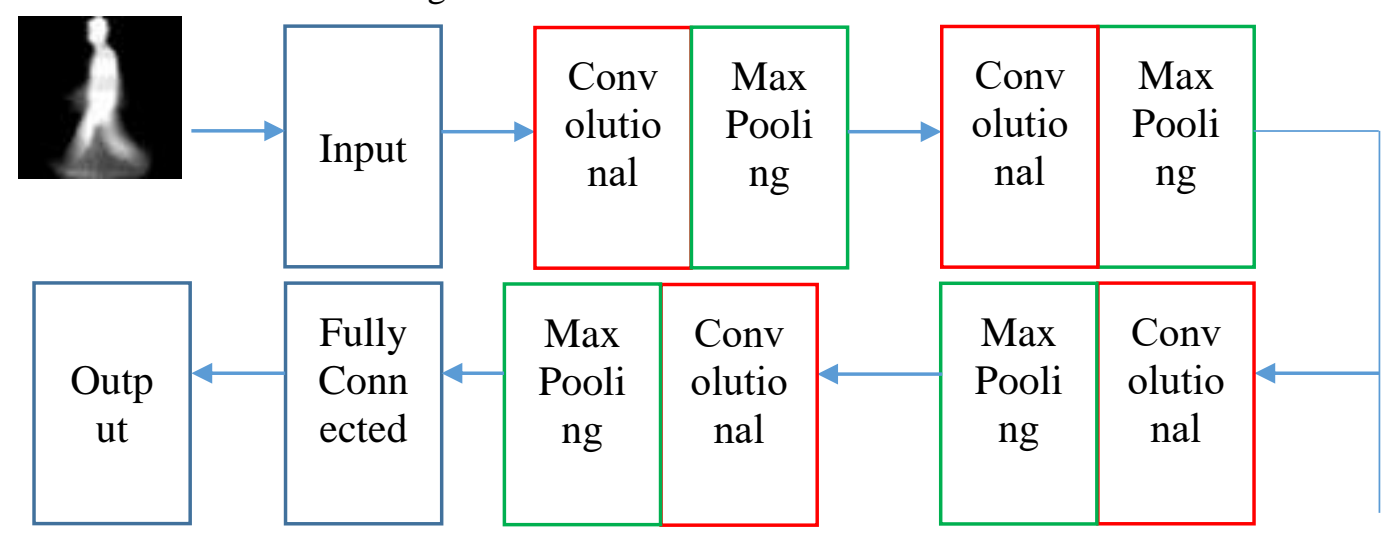

Fig. 1: CNN model by Alotaibi et al.

In the proposed model, a batch standardization process is performed after each convolution pool is conducted. Batch Normalization, similar to normal data standardization, is a way to unify scattered data and optimize neural networks. Data with uniform specifications can make machine learning easier to learn the rules of data. The specific mathematical formula is

$$
\begin{gathered}
Z^{[l]}=W^{[l]} A^{[l-1]}+B^{[l]} \\
\mu=\frac{1}{m} \sum_{i=1}^{m} Z^{[l](i)} \\
\sigma^{2}=\frac{1}{m} \sum_{i=1}^{m}\left(Z^{[l](i)}-\mu\right)^{2} \\
\tilde{Z}^{[l]}=\gamma \cdot \frac{Z^{[l]}-\mu}{\sqrt{\sigma^{2}+\epsilon}}+\beta \\
A^{[l]}=g^{[l]}\left(\tilde{Z}^{[l]}\right) .
\end{gathered}
$$

Batch standardization has the following advantages for data processing: 1. Stabilization of the distribution of each layer of data in the network and acceleration of learning speed. 2. Less sensitivity of parameters in the model multi-network and simplification of the process of adjusting parameters making the learning process more stable. 3. Solving of gradient disappearance problem of network using (sigmoid, tanh, etc.) saturation activation function to a certain extent. 4. Possessing a regularization effect [24]. Combining the advantages of Batch Normalization in data processing, we propose the model shown in Fig. 2. A Batch Normalization process is added to each group of Convolutional layer and Pooling layer. 


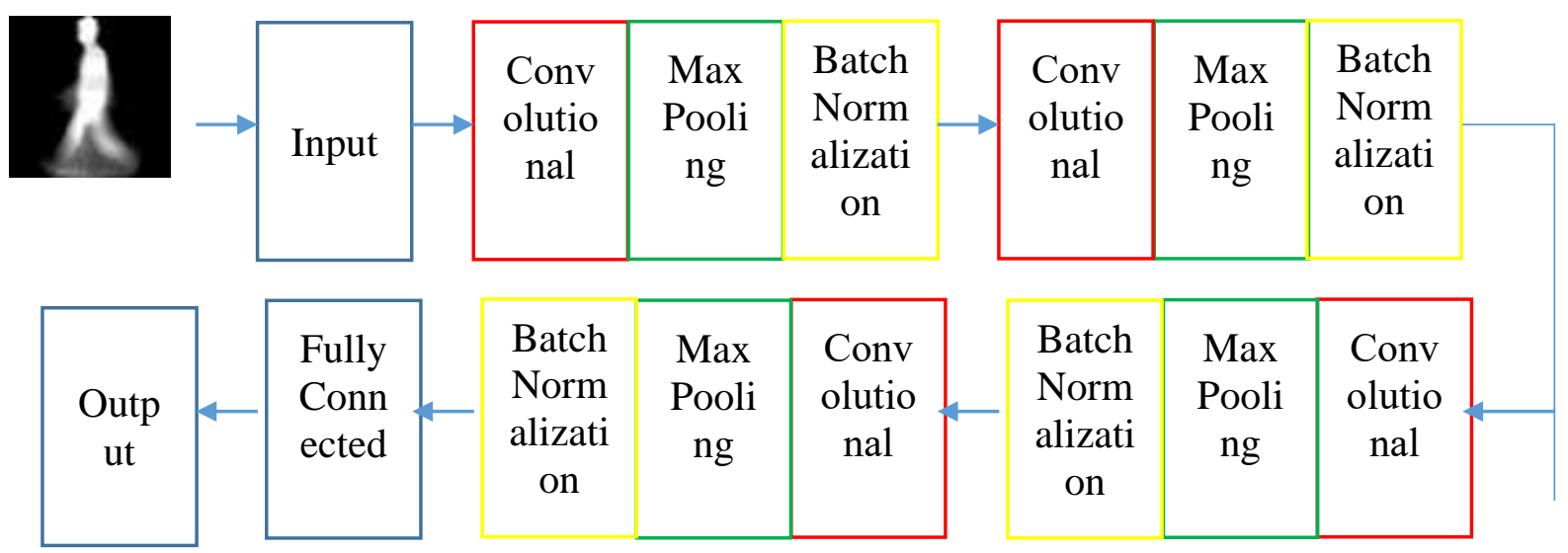

Fig. 2: Proposed CNN model adding Batch Normalization

\subsection{Experimental results and comparison with other gait methods}

The standard dataset used in the comparison experiment is the CASIA Dataset B. Considering the environment in which the subject needs to be applied in practice, only the gait dataset at a single angle of view of 90 degrees is selected for experimentation[25].

The experiment is divided into three groups. The training set of the first group is a series of gait sketches in the case of normal wearing, no outerwear, and no carrying items. In this group, the subjects in test set in data 01-04 under the -nm label are wearing an outerwear. The subjects in test set in data 05-06 under the -nm label are carrying an item. The second set of training sets is a series of gait sketches in the normal wearing without a jacket, and carrying a backpack. The subjects in test set of the -bg label under data 01 have the same wearing, and the subjects in test set of the -bg label under data 02 have the same back bag condition. The training set of the third group is the data of the -cl label with data -01 in the condition of wearing a coat without carrying articles. The test set in this group of the -cl label under data -02 contains subjects wearing a coat without carrying articles. The parameters set in the above three experiments are epoch $=50$ and batch_size $=8$. Experimental results using the proposed model are compared with the results without Batch Normalization. The results are shown in Table 1.

Table 1: Accuracy of experiment under different wearing conditions

\begin{tabular}{|l|l|c|c|}
\hline Methods & \multicolumn{3}{|c|}{ Recognition Accuracy } \\
\hline & nm-nm & bg-bg & cl-cl \\
\hline Deep CNN & 98.3 & 83.87 & 89.12 \\
\hline CNN with Batch normalization & 98.4 & 85.48 & 95.16 \\
\hline
\end{tabular}

Accuracy and loss function are shown in Fig. 3.

From the Table 1 and Fig. 3, it can be seen that the recognition accuracy of the proposed model has improved to some extent, which proves the effectiveness of the model. The proposed model can be used to classify gait silhouette very well, especially in normal wearing without carrying items.

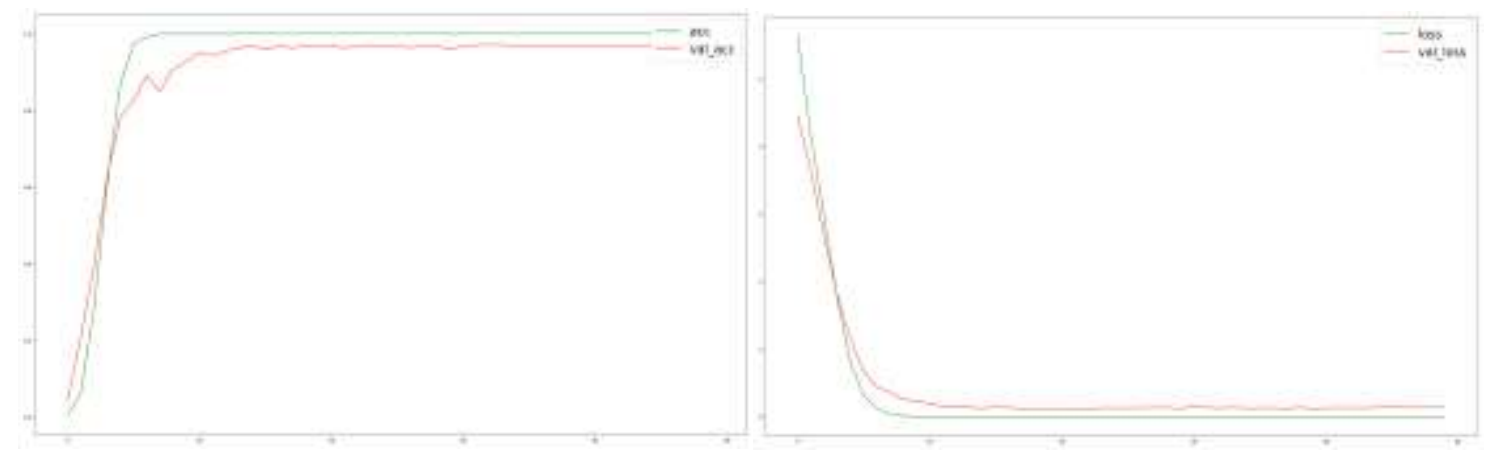

$\mathrm{nm}-\mathrm{nm}$ 


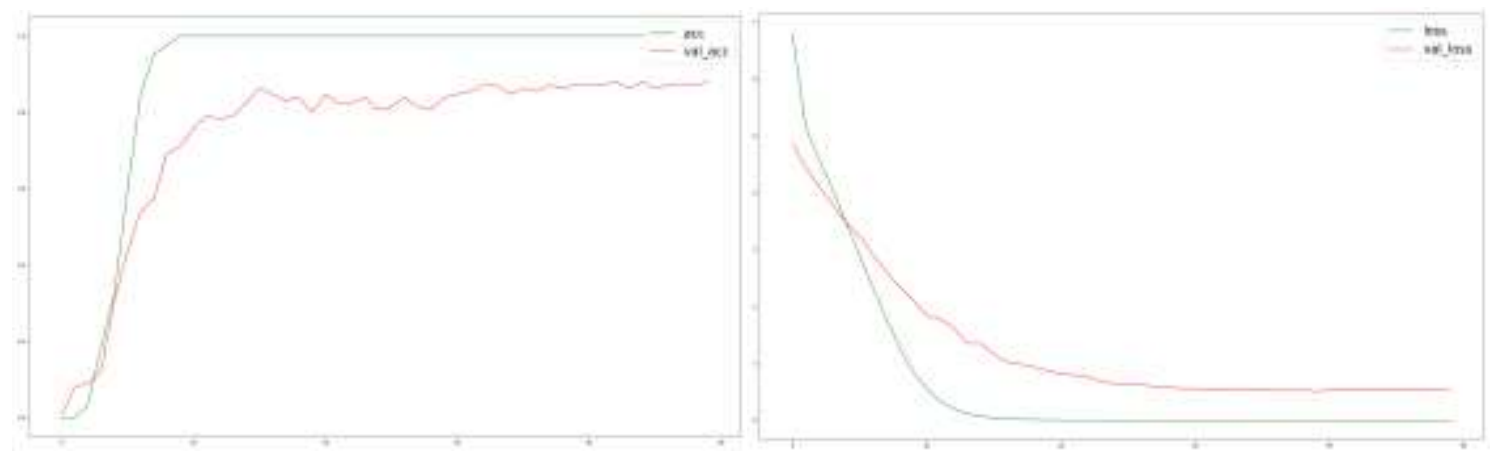

bg-bg

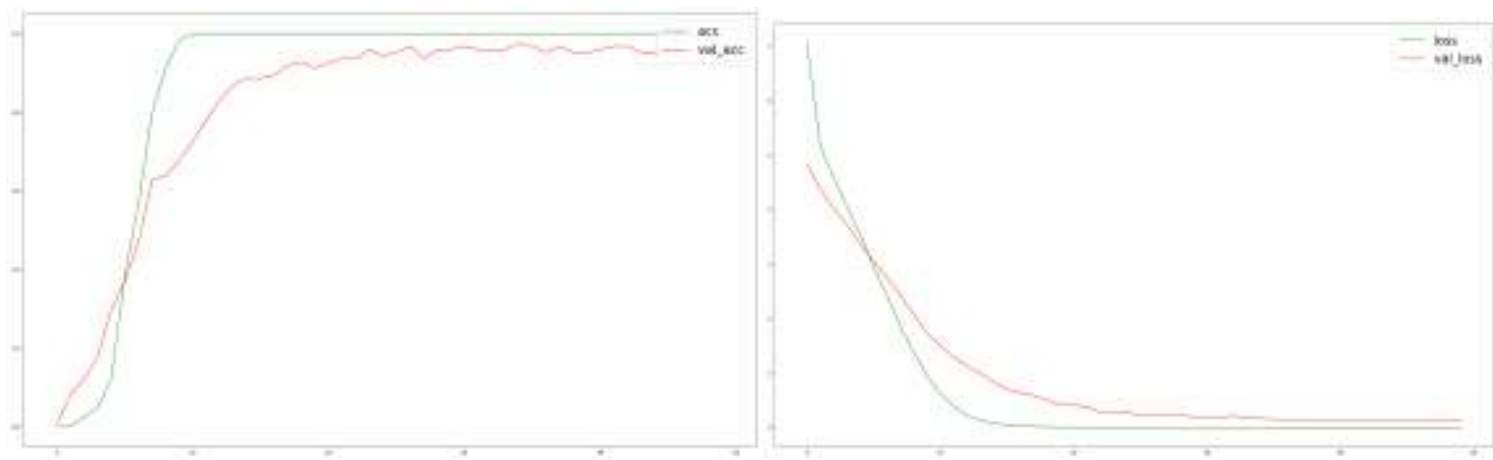

cl-cl

Fig. 3: Accuracy and Loss function.

\section{Construction and Experiment of Own Laboratory Member Gait Dataset}

To construct our own laboratory member gait dataset, we utilize OpenCV to extract pedestrian gait information. A simple test version of the GEI acquisition program interface is shown in Fig. 4.

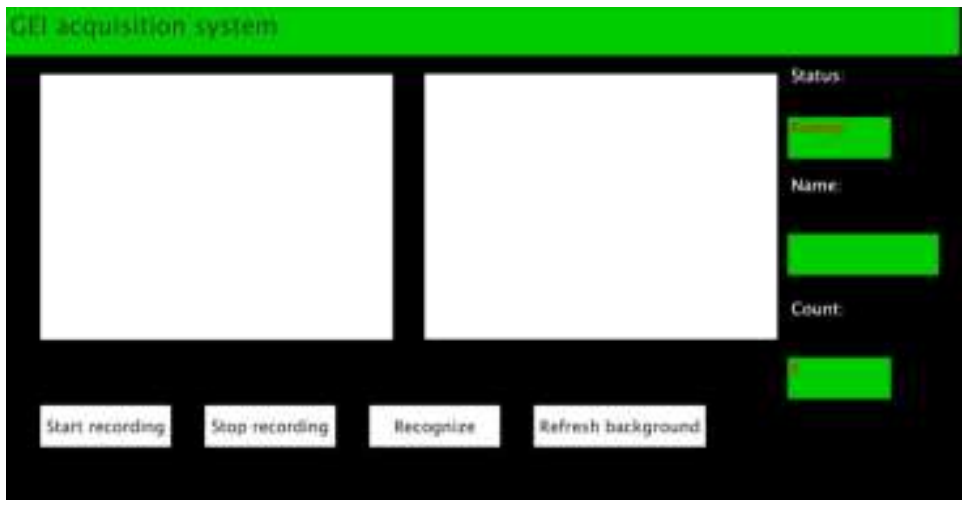

Fig. 4: Obtaining the GEI operation interface Demo

As shown in Fig 7, the interface display is divided into two parts, the left side is the real-time image content captured by the connected camera, and the right side is the mask image after the image is removed from the background. Before acquisition, the Refresh background button is pressed to refresh the background. When there is a pedestrian in front of the camera, the function in OpenCV automatically detects the pedestrian. The Start recording and Stop recording buttons are pressed in turn to record the descendant in a gait. The continuous mask pattern is calculated within the cycle, and according to the GEI acquisition principle, the mask image of each frame is weighted and averaged to obtain the final GEI image. The Recognize button is a function button reserved for later recognition in the interface[26].

During image acquisition, the equipment required and external conditions are as follows. The route between the front of the laboratory and the stairs to be monitored is planned to be the intended road for the subject. After setting the route, a USB camera at a position of 90 degrees is set on the route, so that the information of the acquired GEI is not affected by angle change. During this period, 22 subjects were asked 
to walk from the stairs to the laboratory and from the laboratory to the stairs under normal clothing conditions. During each process, 6 sets of data were acquired. Examples of the data obtained are shown in Fig. 5. Using the data, training and identification of the dataset was done with the proposed model. The parameters were set to epoch $=50$ and batch_size $=8$.

\section{|| || || || || || || || || || || ||}

Fig. 5: Six sets of data at 90 degrees and 270 degrees

\subsection{Experimental results}

This section introduces the process of classifying and identifying the gait dataset of laboratory personnel by using the proposed CNN model. The training and test data were collected using laboratory personnel data focused on the 01-06 label for 90 degrees in CASIA Dataset B. The training set is 01-04 and the test set is 05-06 [14]. The experiment results are shown in Table. 2.

Table 2: Experiment results using test data sets

\begin{tabular}{|l|l|}
\hline Methods & Recognition Accuracy \\
\hline & $\mathrm{nm}-\mathrm{nm}$ \\
\hline CNN with Batch normalization & 88.36 \\
\hline
\end{tabular}

The accuracy and loss function are shown in Fig. 6.

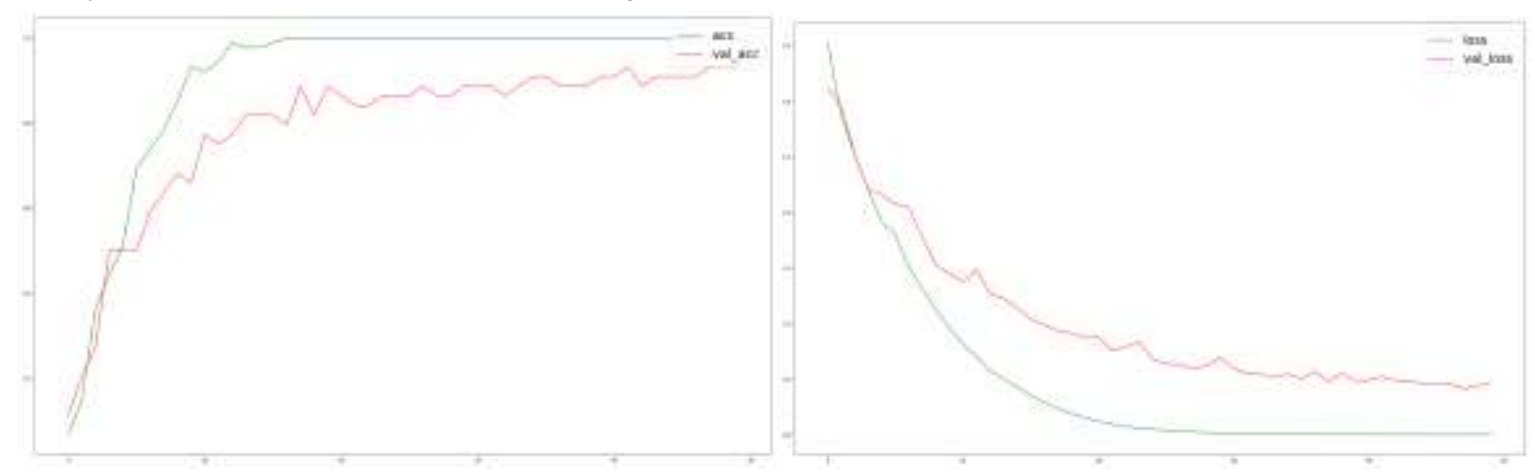

Fig. 6: Accuracy and loss function

The recognition accuracy rate of the proposed method was $88.36 \%$. Due to the small amount of data in the test dataset, this accuracy basically indicates that the gait recognition system proposed in this paper can meet the application requirements. So far, the expectation of using GEI and the proposed Deep CNN model to perform gait recognition to strengthen laboratory safety has been basically achieved.

\subsection{Future work}

In the future, the identification link will be incorporated into the interface, so that the testers in the training set can be visually identified and the applicability can be further enhanced.

\section{Conclusion}

In this paper, we proposed a gait recognition method that incorporates a Batch Normalization process into the CNN to improve recognition accuracy. The GEI dataset in CASIA Dataset B is used for experiment to show the effectiveness of the proposed method. A new dataset constructed by laboratory members also showed a high recognition accuracy rate, implying the capability of the proposed method for actual purposes. The experimental results showed that the proposed model is feasible in the laboratory for safe monitoring. Future work includes further investigation of the model using data obtained from other angles, with improvement and adaptation of the model to general purposes.

\section{Acknowledgments}

This research has been partially supported by JSPS KAKENHI Grant Number $15 \mathrm{H} 01712$. 


\section{References}

[1] Sahani M, Nanda C, Sahu A K, et al. Web-based online embedded door access control and home security system based on face recognition[C]//2015 International Conference on Circuits, Power and Computing Technologies [ICCPCT-2015]. IEEE, 2015: 1-6.

[2] Fuji Ren, Zhong Huang, Automatic facial expression learning method based on humanoid robot XIN-REN, IEEE Transactions on Human-Machine Systems, Vol.46, No.6, pp.810-821,2016

[3] Duo Feng, Shun Nishide and Fuji Ren. Automatic Facial Feature Points Extraction and Expression Recognition Based on Video Database, Proceedings of 4th National Conference on Electrical, Electronics and Computer Engineering, 1525-1530, Dec. 2015.

[4] Reed J H, Gonzalez C R A. Using power fingerprinting (PFP) to monitor the integrity and enhance security of computer based systems: U.S. Patent 9,262,632[P]. 2016-2-16.

[5] Joseph J. Biometric identification and security system associated with cash register: U.S. Patent 6,883,709[P]. 2005-4-26.

[6] Yanqiu Li, Fuji Ren, Lianfeng Xu, Min Hu and Zixi Yu. Face Recognition Based on ULBP and BP Neural Network, the 10th International Conference on Natural Language Processing and Knowledge Engineering, Sapporo, Japan, Oct. 2015.

[7] Yu S, Tan D, Tan T. A framework for evaluating the effect of view angle, clothing and carrying condition on gait recognition[C]//Pattern Recognition, 2006. ICPR 2006. 18th International Conference on. IEEE, 2006, 4: 441-444.

[8] Kim D, Paik J. Gait recognition using active shape model and motion prediction[J]. IET Computer Vision, 2010, 4(1): 25-36.

[9] Haghighat M, Zonouz S, Abdel-Mottaleb M. CloudID: Trustworthy cloud-based and cross-enterprise biometric identification[J]. Expert Systems with Applications, 2015, 42(21): 7905-7916.

[10] Semwal V B, Raj M, Nandi G C. Biometric gait identification based on a multilayer perceptron[J]. Robotics and Autonomous Systems, 2015, 65: 65-75.

[11] Kastaniotis D, Theodorakopoulos I, Theoharatos C, et al. A framework for gait-based recognition using Kinect[J]. Pattern Recognition Letters, 2015, 68: 327-335.

[12] Fuji Ren: From Cloud Computing to Language Engineering,Affective Computing and Advanced Intelligence,International Journal of Advanced Intelligence,Vol.2, No.1,pp.1-14,2010

[13] Kohei Shirage, Yasushi Makihara, Daigo Muramatsu, Tomio Echigo, and Yasushi Yagi,"GEINet: View-invariant gait recognition using a convolutional neural network," in Proceedings of the International Conference on Biometrics, pp. 1-8, 2016.

[14] Alotaibi M, Mahmood A. Improved gait recognition based on specialized deep convolutional neural network [J]. Computer Vision and Image Understanding, 2017, 164: 103-110.

[15] S. Yu, D. Tan, and T. Tan. A framework for evaluating the effect of view angle, clothing and carrying condition on gait recognition. In Proc. of the 18th Int. Conf. on Pattern Recog- nition, volume 4, pages 441-444, Aug. 2006.

[16] Han J, Bhanu B. Individual recognition using gait energy image[J]. IEEE Transactions on Pattern Analysis \& Machine Intelligence, 2006 (2): 316-322.

[17] Preis J, Kessel M, Werner M, et al. Gait recognition with kinect[C]//1st international workshop on kinect in pervasive computing. New Castle, UK, 2012: P1-P4.

[18] Bashir K, Xiang T, Gong S. Feature selection on gait energy image for human identification[C]//Acoustics, Speech and Signal Processing, 2008. ICASSP 2008. IEEE International Conference on. IEEE, 2008: 985-988.

[19] Duo Feng, Fuji Ren. Dynamic Facial Expression Recognition based on Two-Stream-CNN with LBP-TOP, Proceedings of 2018 5th IEEE International Conference on Cloud Computing and Intelligence Systems (CCIS), 355-359, Nanjing, Nov. 2018.

[20] Zhang K, Zuo W, Chen Y, et al. Beyond a gaussian denoiser: Residual learning of deep cnn for image denoising [J] IEEE Transactions on Image Processing, 2017, 26(7): 3142-3155. 
[21] Chen J C, Ranjan R, Sankaranarayanan S, et al. Unconstrained Still/Video-Based Face Verification with Deep Convolutional Neural Networks[J]. International Journal of Computer Vision, 2018, 126(2-4): 272-291.

[22] Yu S, Chen H, Reyes E B G, et al. GaitGAN: Invariant Gait Feature Extraction Using Generative Adversarial Networks[C]//CVPR Workshops. 2017: 532-539.

[23] Takemura N, Makihara Y, Muramatsu D, et al. Multi-view large population gait dataset and its performance evaluation for cross-view gait recognition[J]. IPSJ Transactions on Computer Vision and Applications, 2018, 10(1): 4.

[24] loffe S, Szegedy C. Batch normalization: accelerating deep network training by reducing internal covariate shift[C]// International Conference on International Conference on Machine Learning. JMLR.org, 2015:448-456.

[25] Yu Gu, Jinhai Zhan, Yusheng Ji, Jie Li, Fuji Ren and Shangbin Gao MoSense: An RF-Based Motion Detection System via Off-the-Shelf WiFi Devices, IEEE Internet of Things Journal, Vol.4, No.6, 2326-2341, 2017

[26] Fuji Ren. Affective Information Processing and Recognizing Human Emotion, Electronic Notes in Theoretical Computer Science, Vol.225, No.2009, pp.39-50,2009 